\title{
The association between Vitamin $D$ deficiency and risk of pre-diabetes among older adults
}

\author{
Emily Trussler, Julia Navon, Rachele Pojednic and Elizabeth Metallinos-Katsaras \\ Department of Nutrition, Simmons University, Boston, USA
}

\begin{abstract}
Introduction

Type 2 Diabetes Mellitus is one of the most common conditions affecting older adults, and is one of few diseases with a distinct liminal stage: prediabetes. Dietitians and healthcare practitioners currently recognize prediabetes as a lifestyle disease,and "reversing" it relies largely on dietary modifications and increased exercise.Some research suggests that vitamin D may also be a target since its deficiency has been tentatively linked to many chronic conditions, ${ }^{5}$ and a high prevalence has been observed among subjects with prediabetes and type 2 diabetes. ${ }^{6,7}$ The purpose of this study was to examine the association between vitamin D deficiency and risk of pre-diabetes in people $50+$ years of age living in the United States.
\end{abstract}

Materials and Methods

This was a cross-sectional analysis of the US National Health and Nutrition Examination Survey (2007-2012) subjects, ages 50+ years, free of kidney/liver diseases and diabetes. Outcome: Prediabetes, was defined as: HbA1c level 5.7-6.5\%, or fasting plasma glucose level 100-125 mg/dL, or Oral Glucose Tolerance Test result 140-199 mg/dL, with no laboratory value in the diabetic range. Those with normal glucose tolerance (NGT) with no marker in the prediabetes/diabetes range were the comparison group. Exposure: subjects' vitamin D status was classified based on total serum $25(\mathrm{OH}) \mathrm{D}$ levels as follows: deficient $=<50 \mathrm{nmol} / \mathrm{L}$, insufficient $=50-75 \mathrm{nmol} / \mathrm{L}$, and sufficient $=>75 \mathrm{nmol} / \mathrm{L}$. The final sample $(\mathrm{n}=2,286)$ was sufficient to detect a $7 \%$ difference in odds of prediabetes (the average difference observed in the literature) with $80 \%$ power and $95 \%$ confidence. Multivariate logistic regression analysis included strata, cluster and weight variables. Models were adjusted for BMI, ethnicity, age and gender.

\section{Results}

The final sample was 2,286 subjects, predominantly white $(80.4 \%)$ and female $(56.6 \%)$, with a mean age of 62.3 years; 1,387 had prediabetes $(59.1 \%)$ and 899 were NGT $(40.9 \%)$. Vitamin D status was associated with pre-diabetes $(p=0.03)$. Those with vitamin D deficiency were more likely to have prediabetes compared to vitamin D sufficient individuals (crude OR $=1.4895 \% \mathrm{CI} 1.15-1.91$ ), which remained significant after adjustment for ethnicity, BMI, age and gender ( $\mathrm{aOR}=1.3995 \% \mathrm{CI} 1.02-1.89$ ). There was no effect modification by BMI, gender or ethnicity.

\section{Discussion}

Our results contributed to this existing literature by demonstrating that vitamin D status is associated with pre-diabetes in a nationally representative sample of older US adults.Vitamin D status was significantly associated with risk of prediabetes in Americans $50+$ years of age. Prevention efforts should incorporate awareness of vitamin D.

\section{Conflict of Interest}

There is no conflict of interest 